\title{
Effective Thickness of Laminated Glass Beams. New expression via a variational approach
}

\author{
LAURA GALUPPI \\ Gianni Royer-CARFAGni \\ Department of Civil-Environmental Engineering and Architecture \\ University of Parma, Parco Area delle Scienze 181/A, I 43100 Parma, Italy
}

October 15th 2011

\begin{abstract}
The performance of laminated glass, which consists of two or more glass plies bonded together by polymeric interlayers, depends upon shear coupling between the plies through the polymer. This is commonly considered by defining the effective thickness, i.e., the thickness of a monolithic beam with equivalent bending properties in terms of stress and deflection. General expressions have been proposed on the basis of simplified models by Newmark and Wölfel-Bennison, but they are either difficult to apply or inaccurate. Here, a variational approach to the problem is presented. By choosing appropriate shape functions for the laminated-beam deformation, minimization of the strain energy functional gives new expressions for the effective thickness under any constraint- and load-conditions, embracing the classical formulations as particular cases. Comparisons with numerical experiments confirm the better accuracy of the proposed approach with respect to the previous ones.
\end{abstract}

KEYWORDs: Structural glass, laminated glass, composite structures, bending strength, effective thickness, variational approach.

\section{Introduction}

In order to reduce the risk of catastrophic collapse of structures made of glass, the brittle material par excellence, an effective technique is to bond two or more glass plies with 
thermoplastic polymeric interlayers with a treatment in autoclave at high pressure and temperature. This bond is quite strong because it is chemical in type, being due to the union between hydroxyl groups along the polymer and silanol groups on the glass surface. The resulting laminated glass is a safety glass because, after breakage, the fragments remain attached to the interlayer: risk of injuries is reduced and the element maintains a certain consistency that prevents detachment from fixings. But the iterlayer affects also the pre glass-breakage response because it allows the transfer of shear stresses among glass plies, at the price of a relative sliding due to the deformation of the polymer. The assessment of the degree of connection offered by the polymer is crucial for the design of glass structures in the serviceability limit state and this is why a great number of studies, including this one, have considered the response of the composite laminated package before first cracking occurs.

Indeed, the polymeric interlayers are too soft to present flexural stiffness per se, but they can provide shear stresses that play an important role for the glass-layer interaction [7]. In general, the degree of coupling of two glass layers depends upon the shear stiffness of the polymeric interlayer, as first mentioned by Hooper in 1973 [3] while studying the bending of simply supported laminated-glass beams. Since then, the problem has been considered by many authors [15], one of the most recent contribution being the careful finite element analysis of [14], which includes an updated list of the most relevant literature.

In the pre glass-breakage modeling no distinction has to be made for what the type of glass is concerned, because all treatments (annealing, heat strengthening, heat or chemical tempering) affect the ultimate strength and the type of rupture (size of resulting shards) but not the elastic moduli (Young's modulus $E \simeq 70 G P a$ and Poisson ratio $\nu \simeq 0.2$ ). If the response of glass is linear elastic up to failure, the response of the polymeric interlayer is highly non-linear, temperature-dependent and viscoelastic. There are three main commercial polymeric films, each one showing peculiar characteristics: Polyvinyl Butyral (PVB), Ethylene Vinyl Acetate (EVA), and Sentry Glass (SG) [16] [10]. Pure PVB, a polyvinyl acetate, is stiff and brittle, but addition of softeners imparts plasticity and toughness, though influencing adhesion-strength, elasticity, water-absorbing and dependence on temperature (glass transition temperature $T_{g}$ of the order of $20-25^{\circ} \mathrm{C}$ ). Depending on the composition, the properties of EVA, a polyolefine, vary from partial crystalline and thermoplastic to amorphous and rubber-like, but an increased quantity of vinyl acetate improves strength and ultimate elongation, though decreasing melting temperature: when used as interlayers in laminated glass, modified EVAs are employed with mechanical properties similar to PVB. A somehow innovative material is SG, a ionoplast polymer that, when compared with PVB, presents higher stiffness $(>100 \times \mathrm{PVB})$, strength $(>5 \times \mathrm{PVB})$, resistance to temperature $\left(T_{g} \sim 60^{\circ} \mathrm{C}\right)$. Depending upon polymer type, room-temperature $T$ and characteristic loadduration $t_{0}$, the secant shear modulus of the interlayer may vary from $0.01 \mathrm{Mpa}$ (PVB at $T=+60^{\circ} \mathrm{C}$ under permanent load) up to $300 \mathrm{MPa}$ (SG at $T=0^{\circ} \mathrm{C}$ and $t_{0}=1 \mathrm{sec}$ ).

As pointed out by Norville [8], the response of the laminated glass beams may vary between two borderline cases: i) the layered limit, when the beam is composed of free-sliding 
glass plies; ii) the monolithic limit, where no relative slippage occurs and the classical EulerBernoulli assumption that "plane sections remain plane" does hold. In the layered limit, the various plies equilibrate, in parallel, an aliquot of the applied load proportional to their bending stiffness; in the monolithic limit, the response of the composite beam approaches that of a homogeneous beam with equal cross-section. It must be mentioned that in most models the interlayer thickness is not explicitly considered: ignoring the spacing provided by the polymeric layers, the equivalent monolith is often taken to be one whose thickness equals the sum of that of the glass plies. Experimental tests, however, show [9] that the laminate can be stronger than this. Indeed, the monolithic limit has to be associated with the case of a beam for which the cross-sectional inertia equals that of the cross sections of the composing glass layers, properly spaced of the interlayer gaps.

The capability of the interlayer to transfer shear stress between each pair of connected glass surfaces is affected by load-level, load-duration, temperature, adhesion properties. This dependence has been investigated in several studies [7, 13, 19], but consideration of all these effects in modelling would be very complicated. A quite-effective technical solution, widely employed in the design practice, consists in assuming perfect glass-polymer adhesion and in considering the polymer as a linear elastic material, characterized by proper secant elastic moduli calibrated according to room temperature and load duration. Geometric non-linearities are usually important because of the slenderness of the laminated panel [11], but can be neglected, at least as a first order approximation, when the loads are mainly orthogonal to the panel surface and no in-plane forces are present.

Numerical 3D models are used for the most important works only, while 2D or 1D models are usually preferred because of their simplicity. These are based upon the definition of an "equivalent" beam or plate whose effective thickness is properly downgraded when compared with the monolithic limit. In simple words, the effective thickness of a laminated glass plate is the (constant) thickness of the homogeneous plate that, under the same boundary and load conditions of the problem at hand, presents the same maximal stress or maximal deflection. This definition is very practical, but in the technical literature and in the national standards various formulations that lead to diverse expressions for the equivalent thickness have been proposed. In substance, the two most common types of models are the one derived from the theory by Newmark [2] for layered composite beams with deformable connectors and that proposed by Bennison et al. [17] [10] based upon the original approach for sandwich beams by Wölfel [4]. Both formulations furnish a one-dimensional analysis of the laminated-glass beam under the hypothesis that the interlayer adhesion is sufficient to keep constant the relative distance between the bonded glass surfaces, although their relative slippage may be allowed by the relatively-low shear stiffness of the polymer.

The aim of this paper is to revise the classical problem of a composite laminated glass beam under flexure, assuming linear elastic response for the components (glass + polymer) and ruling out delamination and geometric non-linearities. A variational approach to this problem is presented that automatically furnishes the governing differential equations and boundary conditions. To this respect, the approach is substantially similar to that proposed 
in [12] for numerical purposes, but here it is specialized towards two major goals: $i$ ) the comparison of the various existing models under a common variation framework; ii) the definition of more accurate expressions for the effective thickness that may apply to a large number of constraint and load conditions. In particular, using convenient shape functions that fulfills general requirements of the solution, we find a simplified formulation, original to our knowledge, that can be used to define new expressions of the equivalent thickness for the laminated package. Comparisons with the traditional formulations and with numerical experiments based upon refined FEM models, highlight the accuracy of the proposed approach with respect to previous ones, especially under boundary and load conditions that are different from the commonly considered case of simply-supported beams under uniform loading.

\section{Review of simple models for laminated glass.}

The classical expressions for the effective thickness of laminated glass rely upon simplifying assumptions in modeling that are hardly mentioned in the literature. A brief review that will be useful for the forthcoming considerations is here provided.

\section{$2.1 \quad$ Newmark's model.}

A simple analytical model for a structure made of two beams with elastic interaction was proposed in 1951 by Newmark [2]. This theory was originally conceived of for a typical steel-concrete composite bridge deck, formed by a concrete slab and a steel beam bonded by shear connectors. The assumptions of the model are the following: $i$ ) the shear connection is continuous; $i i$ ) the amount of slip permitted by the shear connection is directly proportional to the load transmitted; iii) the strain distribution in the slab and in the beam is linear; $i v$ ) the slab and the beam deflect equal amounts at all points along their length. The model is applicable to any composite beam consisting of two elements with bending stiffness, connected by an interface with negligible thickness that transfers shear forces. Therefore, it can be conveniently used for laminated glass.

Consider, as indicated in figure 1, a laminated beam of length $l$ and width $b$ composed of two glass plies of thickness $h_{1}$ and $h_{2}$ and Young's modulus $E$, connected by a polymeric interlayer of thickness $t$ and shear modulus $G$. Let

$A_{i}=h_{i} b, I_{i}=\frac{b h_{i}^{3}}{12}(i=1,2), H=t+\frac{h_{1}+h_{2}}{2}, A^{*}=\frac{A_{1} A_{2}}{A_{1}+A_{2}}, I_{t o t}=I_{1}+I_{2}+A^{*} H^{2}, A=b t$ 

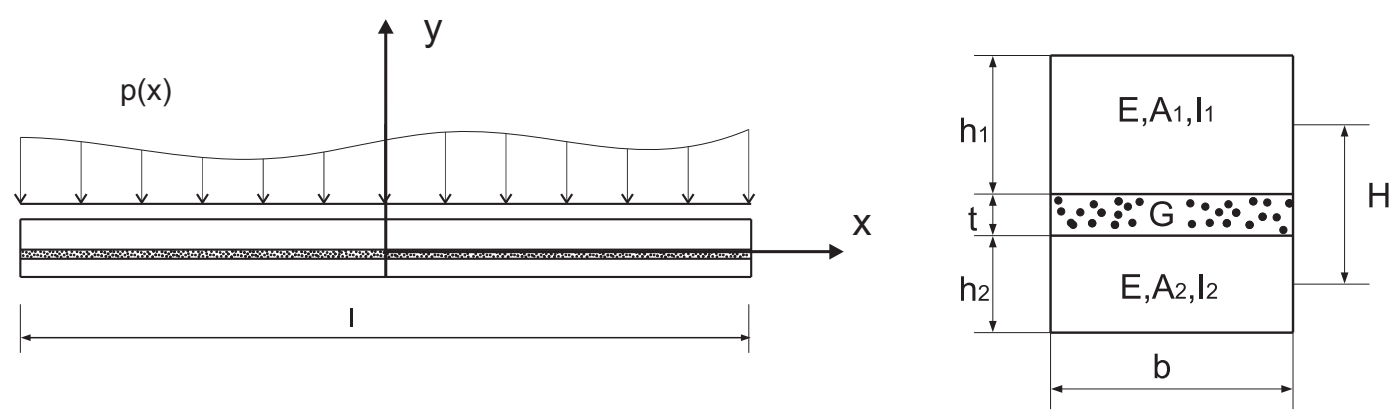

Figure 1: Beam composed of two glass plies bonded by a polymeric interlayer. Longitudinal and cross sectional view (not in the same scale).

and observe, in particular, that $I_{\text {tot }}$ represents the moment of inertia of the full composite section (monolithic limit) that takes into account the spacing provided by the interlayer gap. Introduced a right-handed orthogonal reference frame $(x, y)$ with $x$ parallel to the beam axis, supposed horizontal, and $y$ directed upwards, the transversal displacement $v(x)$ is positive if in the same direction of increasing $y$, the transversal load $p(x)>0$ if directed downwards, while the bending moment $M(x)$ is such that $M^{\prime \prime}(x)>0$ when $v^{\prime \prime}(x)>0$. Then, as shown in [6], $v(x)$ has to satisfy the differential equation

$$
v^{\prime \prime \prime \prime}(x)-\alpha^{2} v^{\prime \prime}(x)+\alpha^{2} \frac{M(x)}{E I_{\text {tot }}}+\frac{p(x)}{E\left(I_{1}+I_{2}\right)}=0,
$$

where

$$
K=\frac{G b}{t}, \alpha^{2}=\frac{K}{E A^{*}} \frac{I_{t o t}}{I_{1}+I_{2}} .
$$

The model is accurate, but the solution of the differential equation (2.2) is relatively simple only if the bending moment $M(x)$ is known, i.e., the beam is statically determinate. Moreover, the model is clearly one-dimensional and cannot be extrapolated to the case of plates or shells under general boundary conditions.

\subsection{The approach by Wölfel-Bennison.}

The modeling that relies upon the original approach by Wölfel for composite sandwich structures is attractive because of its simplicity, so to be mentioned in many structural standards. In fact, it provides a direct method to calculate the "effective thickness" of a monolithic beam with equivalent bending properties to a laminated beam. However, this approach relies upon several simplifying assumption that it is necessary to recall here, because they are useful to understand the limits of the theory but, to our knowledge, they 
are never mentioned in the technical literature, apart from the original work by Wölfel [4] that is difficult to find and written in German.

The model was primarily conceived of for a sandwich structure composed of three layers, the external ones with considerable axial stiffness but negligible bending stiffness, while the intermediate layer can only bear shear stress with zero axial and flexural strength. Therefore, it well applies to sandwich panels composed of, e.g., a soft core and external metallic coating layers. With the same notation of (2.1), let $M(x)$ and $\bar{M}(x)$, represent the bending moments in the beam under, respectively, the external service load $q(x)$ and a concentrated unit force at midspan, while $Q(x)$ and $\bar{Q}(x)$ denote the corresponding shear forces. If $\chi$ is the shear coefficient of the intermediate (soft) layer, from the principle of virtual work the sag $\delta$ at midspan turns out to be:

$$
\begin{array}{r}
\delta=\frac{1}{B_{s}} \int_{0}^{l} M(x) \bar{M}(x) d x+\frac{\chi}{G A} \int_{0}^{l} Q(x) \bar{Q}(x) d x=\frac{(1+\mathcal{K})}{B_{s}} \int_{0}^{l} M(x) \bar{M}(x) d x \\
\mathcal{K}=\beta B_{s} \frac{\chi}{G A l^{2}}, \quad \beta=\frac{l^{2} \int_{0}^{l} Q(x) \bar{Q}(x) d x}{\int_{0}^{l} M(x) \bar{M}(x) d x},
\end{array}
$$

where $B_{s}=E A^{*} H^{2}$ represents the bending stiffness of the two areas $A_{1}$ and $A_{2}$, supposed to be concentrated in the corresponding centroid and thus neglecting their individual bending stiffness. The coefficient $\beta$ in $(2.4)_{3}$ depends upon the load condition and, for the most common cases, the corresponding values are recorded in [4]. In particular: $\beta=9.6$ when the load is uniformly distributed; $\beta=12$ for a concentrated force at midspan; $\beta=\pi^{2}$ for a sinusoidal load.

When the external layers presents considerable bending stiffness, Wölfel proposed an approximate solution according to which the bending stiffness $B_{s}$ in (2.4) should be substituted by $B_{s}^{*}$ defined as

$$
\delta=\frac{1}{B_{s}^{*}} \int_{0}^{l} M(x) \bar{M}(x) d x, B_{s}^{*}=E I_{1}+E I_{2}+\frac{1}{1+\mathcal{K}} B_{s}
$$

that assumes that the individual bending stiffness of the external layers has no influence on the bonding offered by the central layer: the less the bending stiffness of the external layers, the more accurate is this hypothesis. Notice from (2.4) that $\mathcal{K} \in(0,+\infty)$. In particular, $G \rightarrow \infty \Rightarrow \mathcal{K} \rightarrow 0$, so that from (2.5) also $B_{s}^{*} \rightarrow E I_{\text {tot }}$ (monolithic limit); moreover, 
$G \rightarrow 0 \Rightarrow \mathcal{K} \rightarrow \infty$ and $B_{s}^{*} \rightarrow E\left(I_{1}+I_{2}\right)$ (layered limit). Henceforth, the coefficient $(1+\mathcal{K})$ indicates the degradation of the bending stiffness due to the incomplete interaction between the external layers.

Bennison et al. [17, 18] have developed Wölfel's approach specifically for the case of laminated glass, by proposing to use (2.5) to calculate the deflection of a laminated glass beam. More precisely, the authors call $\Gamma=1 /(1+\mathcal{K}), \Gamma \in(0,1)$, and introduce the equivalent moment of inertia of the cross section in the form

$$
I_{e q}=I_{1}+I_{2}+\Gamma \frac{A_{1} A_{2}}{A_{1}+A_{2}} H^{2}
$$

In order to evaluate the shear coefficient $\chi$ that takes part in the definition of $\mathcal{K}$ and, consequently, of $\Gamma$, it is necessary to estimate the shear stresses $\tau$ and $\bar{\tau}$, associated with the shear forces $Q(x)$ and $\bar{Q}(x)$, respectively. Under the same simplifying hypotheses that had led to (2.4), i.e., neglecting the flexural inertia of each one of the external plies, one finds that $\tau b=Q / H$ and $\bar{\tau} b=\bar{Q} / H$. Then the shear coefficient, which is defined by

$$
\frac{\chi}{G A} \int_{0}^{l} Q \bar{Q} d x=\frac{1}{G} \int_{0}^{l} \int_{A} \tau \bar{\tau} d A d x=\frac{1}{G} \int_{0}^{l} \int_{A} \frac{Q \bar{Q}}{(H b)^{2}} d A d x=\frac{A}{G(H b)^{2}} \int_{0}^{l} Q \bar{Q} d x
$$

turns out to be

$$
\chi=\frac{A^{2}}{(H b)^{2}}=\frac{t^{2}}{H^{2}}
$$

The proposal by Bennison et al. is to consider for $\Gamma$ the universal expression

$$
\Gamma=\frac{1}{1+\beta \frac{\chi B_{s}}{G A l^{2}}}=\frac{1}{1+9.6 \frac{t B_{s}}{G b H^{2} l^{2}}}
$$

with $\beta=9.6$ as in the case of uniformly distributed loading. More precisely, the authors define the nominal values $h_{s ; 1}$ and $h_{s ; 2}$ of the thickness and the "bonding inertia" $I_{s}$

$$
h_{s ; 1}=\frac{H h_{1}}{h_{1}+h_{2}}, h_{s ; 2}=\frac{H h_{2}}{h_{1}+h_{2}}, \quad I_{s}=\frac{B_{s}}{E b}=\frac{h_{1} h_{2}}{h_{1}+h_{2}} H^{2}=h_{1} h_{s ; 2}^{2}+h_{2} h_{s ; 1}^{2} .
$$

so that $\Gamma$ of $(2.9)$ can be written as 


$$
\Gamma=\frac{1}{1+9.6 \frac{E I_{s} t}{G H^{2} l^{2}}} .
$$

Consequently, recalling (2.6), for calculating the laminate deflection one can consider a monolithic beam with deflection-effective thickness $h_{\text {ef; }}$ given by

$$
h_{e f ; w}=\sqrt[3]{h_{1}^{3}+h_{2}^{3}+12 \Gamma I_{s}} .
$$

Once the effective stiffness of the laminate is established, the maximum stress in the glass can be easily estimated. Eventually, one finds that the maximum bending stress in each glass plies is the same of that in a fictitious monolithic beam loaded by the service load $q(x)$ with respectively stress-effective thickness

$$
h_{1 ; e f ; \sigma}=\sqrt{\frac{h_{e f ; w}^{3}}{h_{1}+2 \Gamma h_{s ; 2}}}, h_{2 ; e f ; \sigma}=\sqrt{\frac{h_{e f ; w}^{3}}{h_{2}+2 \Gamma h_{s ; 1}}} .
$$

It is important to notice that the method of the effective thickness relies upon the assumed form of $\Gamma$ given by (2.9) and (2.11), which contains the coefficient $\beta=9.6$, i.e., the one proposed by Wölfel for the unique case of simply supported beams under uniformly distributed loading. For other kind of loading and boundary constraint, other values for $\beta$ should be chosen. Moreover, according to Wölfel himself [4], the validity of the method is limited because its simplifying assumptions are valid for statically-determined composite beams, for which the bending stiffness of the composite plies is negligible.

\section{The energetic approach}

With respect to a reference system $(x, y)$ as in fig. 1 , consider the laminated beam $-l / 2 \leq$ $x \leq l / 2$ composed of two glass plies bonded by a thin polymeric interlayer, whose geometric sizes are defined by the same parameters of (2.1). Again, $E$ and $G$ represent the Young's modulus of the glass and the shear modulus of the interlayer, and the beam is loaded under a generic load per unit length $p(x)$, not necessarily uniformly distributed.

\subsection{The variational formulation.}

The glass-polymer bond is supposed to be perfect and the interlayer strain in direction $y$ is negligible. Under the hypothesis that strains are small and the rotations moderate, the kinematics is completely described by the vertical displacement $v(x)$, the same for the two 

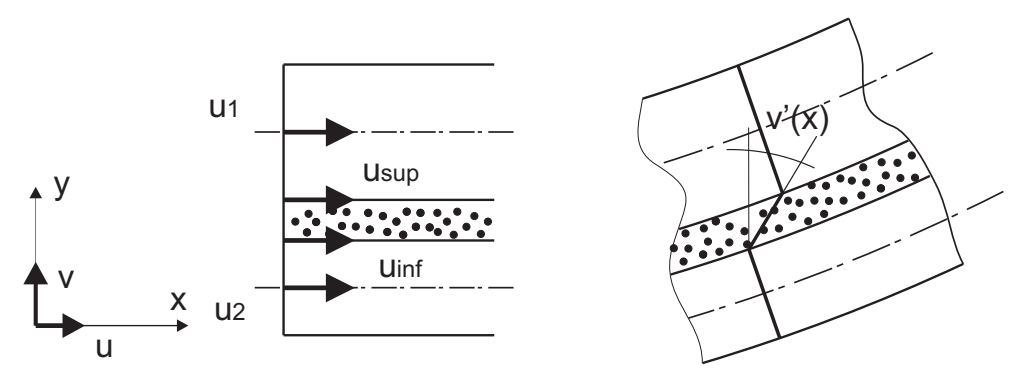

Figure 2: Relevant displacement components and corresponding deformation in the composite beam

glass components, and the horizontal displacements $u_{1}(x)$ and $u_{2}(x)$ of the centroid of the upper and lower glass element, respectively. As shown in detail in figure 2 , let $u_{\text {sup }}$ and $u_{\text {inf }}$ denote the horizontal displacement at the intrados of the upper glass element and the extrados of the lower glass element, i.e., at the interface with the interlayer. Then, the shear strain in the interlayer is then given by

$$
\gamma=\frac{1}{t}\left[u_{\text {sup }}-u_{\text {inf }}+v^{\prime}(x) t\right]=\frac{1}{t}\left[u_{1}(x)-u_{2}(x)+v^{\prime}(x) H\right] .
$$

The corresponding energy of the system ([1], Chap. III) may be written as follows:

$$
\begin{aligned}
& \mathfrak{E}\left[u_{1}(x), u_{2}(x), v(x)\right]= \\
& \int_{-l / 2}^{l / 2}\left\{\frac{1}{2}\left[E\left(I_{1}+I_{2}\right) v^{\prime \prime}(x)^{2}+E A_{1} u_{1}^{\prime}(x)^{2}+E A_{2} u_{2}^{\prime}(x)^{2}+\frac{G b}{t}\left(u_{1}(x)-u_{2}(x)+v^{\prime}(x) H\right)^{2}\right]+p v(x)\right\} d x .
\end{aligned}
$$

where the first term represents the flexional contributions, the second and the third terms are the extensional strain energy of the upper and lower glass plies, respectively, whereas the fourth term corresponds to the interfacial strain energy due to the shear elastic strain of the polymer; the last term expresses the contribution of the loading $p(x)$. The zeroing of the first variation with respect to $v(x), u_{1}(x)$ and $u_{2}(x)$ gives respectively the Euler's equilibrium equations

$$
\begin{gathered}
E\left(I_{1}+I_{2}\right) v^{\prime \prime \prime \prime}(x)-\frac{G b}{t}\left(u_{1}(x)-u_{2}(x)+v^{\prime}(x) H\right)^{\prime} H+p=0, \\
E A_{1} u_{1}^{\prime \prime}(x)=\frac{G b}{t}\left(u_{1}(x)-u_{2}(x)+v^{\prime}(x) H\right), \\
E A_{2} u_{2}^{\prime \prime}(x)=-\frac{G b}{t}\left(u_{1}(x)-u_{2}(x)+v^{\prime}(x) H\right) .
\end{gathered}
$$


Observe, in passing, that equations (3.4) and (3.5) can be rearranged as

$$
\begin{gathered}
A_{1} u_{1}^{\prime \prime}(x)=-A_{2} u_{2}^{\prime \prime}(x), \\
\frac{G b}{t}\left(u_{1}(x)-u_{2}(x)+v^{\prime}(x) H\right)=\frac{E}{2}\left[A_{1} u_{1}^{\prime \prime}(x)-A_{2} u_{2}^{\prime \prime}(x)\right] .
\end{gathered}
$$

Recalling that $E A_{i} u_{i}^{\prime \prime}(x)$ is the derivative of the axial force $N_{i}$ in the $i$ - th glass layer, conditions (3.6) and (3.7) represent the axial equilibrium of the two glass plies under the mutual shear force per unit length $\tau$, transmitted by the polymeric interlayer (fig. $3 a$ ), i.e., $A_{1} u_{1}^{\prime \prime}(x)=-A_{2} u_{2}^{\prime \prime}(x)=\tau=G \gamma b$. Such shear contributions are statically equipollent to a distributed torque per unit length equal to $-\tau\left(h_{1} / 2+t^{*}\right)$ ( $t^{*}$ is arbitrary as shown in figure 3$)$ in the upper glass beam, $-\tau\left(h_{2} / 2+t-t^{*}\right)$ in the lower glass beam and $-\tau t$ in the interlayer. Then, as represented in fig. $3 b$, condition (3.3) represents the equilibrium under bending of the package glass + polymer, which is of the form $E I v^{\prime \prime \prime \prime}(x)+p(x)+m^{\prime}(x)=0$, with $I=I_{1}+I_{2}$ and $m(x)=-\tau(x)\left(\frac{h_{1}+h_{2}}{2}+t\right)=-\tau(x) H$.
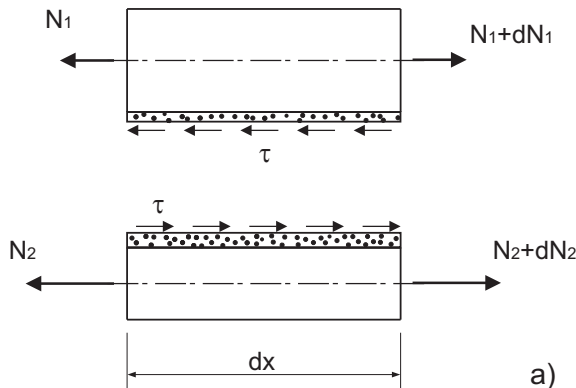

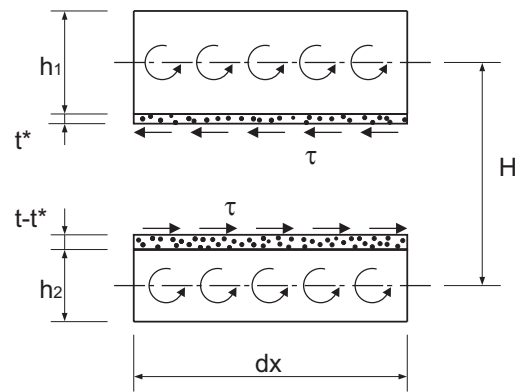

b)

Figure 3: Interpretation of the Euler's equations in terms of equilibrium of an infinitesimal voussoir.

Standard arguments in the calculus of variation [5] furnish the boundary conditions

$$
\begin{aligned}
& {\left[-\left(E\left(I_{1}+I_{2}\right) v^{\prime \prime \prime}(x)+\frac{G b}{t} \gamma(x) H\right) \delta v(x)\right]_{-l / 2}^{l / 2}=0,} \\
& {\left[E\left(I_{1}+I_{2}\right) v^{\prime \prime}(x) \delta v^{\prime}(x)\right]_{-l / 2}^{l / 2}=0,} \\
& {\left[E A_{1} u_{1}^{\prime}(x) \delta u_{1}(x)\right]_{-l / 2}^{l / 2}=0,} \\
& {\left[E A_{2} u_{2}^{\prime}(x) \delta u_{2}(x)\right]_{-l / 2}^{l / 2}=0,}
\end{aligned}
$$

where $\delta v(x), \delta u_{1}(x)$ and $\delta u_{2}(x)$ denote the variations of $v(x), u_{1}(x)$ and $u_{2}(x)$. Such variations are null at the boundary where the displacement is prescribed, arbitrary otherwise. 
It should be also observed that whenever $G \rightarrow 0$, the Euler's equation give

$$
\left\{\begin{array}{l}
E\left(I_{1}+I_{2}\right) v^{\prime \prime \prime \prime}(x)+p=0 \\
E A_{1} u_{1}^{\prime \prime}(x)=0 \\
E A_{2} u_{2}^{\prime \prime}(x)=0
\end{array}\right.
$$

that correspond to the the equilibrium of two frictionless sliding glass beams (layered limit).

In order to recover the equilibrium equation for the case of perfect bonding, a relationship between the horizontal displacement of the upper and lower glass plies can be found from equation (3.6). Observe that such an equation can be rearranged in the form:

$$
\left(E A_{1} u_{1}^{\prime}(x)+E A_{2} u_{2}^{\prime}(x)\right)^{\prime}=0 \quad \Rightarrow \quad E A_{1} u_{1}^{\prime}(x)+E A_{2} u_{2}^{\prime}(x)=\text { const },
$$

where $E A_{1} u_{1}^{\prime}(x)=N_{1}(x)$ and $E A_{2} u_{2}^{\prime}(x)=N_{2}(x)$ represent the axial forces in the glass layers at $x$. If the beam is not constrained at one of its ends, so that $\delta u_{1}(l / 2) \neq 0$ and $\delta u_{2}(l / 2) \neq 0\left(\right.$ or $\delta u_{1}(-l / 2) \neq 0$ and $\left.\delta u_{2}(-l / 2) \neq 0\right)$, then $E A_{1} u_{1}^{\prime}(l / 2)=E A_{2} u_{2}^{\prime}(l / 2)=0$ (or $E A_{1} u_{1}^{\prime}(-l / 2)=E A_{2} u_{2}^{\prime}(-l / 2)=0$ ) and, consequently, $E A_{1} u_{1}^{\prime}(l / 2)+E A_{2} u_{2}^{\prime}(l / 2)=0$ (or $E A_{1} u_{1}^{\prime}(-l / 2)+E A_{2} u_{2}^{\prime}(-l / 2)=0$ ). From (3.10), this leads to $N_{1}(x)+N_{2}(x)=0$, $\forall x \in(-l / 2, l / 2)$.

In the most general case in which the beam is constrained at both its ends so that $\delta u_{1}( \pm l / 2)=\delta u_{2}( \pm l / 2)=0$, we may suppose that no axial elongation is given to each glass ply, i.e.,

$$
\int_{-l / 2}^{l / 2} u_{1}^{\prime}(x) d x=\int_{-l / 2}^{l / 2} u_{2}^{\prime}(x) d x=0 .
$$

Then, if one assumed that $N_{1}(x)+N_{2}(x)=N \neq 0$, where $N$ represents the resultant axial force in the composite beam, one would find

$$
0=\int_{-l / 2}^{l / 2}\left(E A_{1} u_{1}^{\prime}(x)+E A_{2} u_{2}^{\prime}(x)\right) d x=N l \quad \Rightarrow \quad N=0 .
$$

In conclusion, if one of the bar ends is not constrained, or if the bar ends are not displaced apart, the axial resultant force is null, leading to:

$$
\left(E A_{1} u_{1}(x)+E A_{2} u_{2}(x)\right)^{\prime}=0 \quad \Rightarrow \quad A_{1} u_{1}(x)+A_{2} u_{2}(x)=\text { const },
$$

In order to prevent the rigid body motion in $x$ direction, at least one point of the structure may be rigidly fixed in space. This condition may be imposed not only on the displacement of 
the upper or lower glass ply, but also on the (weighted) average displacement field $A_{1} u_{1}(x)+$ $A_{2} u_{2}(x)$, leading to

$$
A_{1} u_{1}(x)+A_{2} u_{2}(x)=0 \quad \Rightarrow \quad u_{2}(x)=-\frac{A_{1}}{A_{2}} u_{1}(x) .
$$

Equation (3.14) in the case of perfect bonding, i.e. $G \rightarrow \infty, \gamma=0$, leads to the following relationship between vertical and horizontal displacement:

$$
\gamma=u_{1}(x)-u_{2}(x)+v^{\prime}(x) H=0 \quad \rightarrow \quad u_{1}(x)=-v^{\prime}(x) H \frac{A_{2}}{A_{1}+A_{2}},
$$

leading to

$$
G b \gamma=E A_{1} u_{1}(x)=-E H A^{*} v^{\prime \prime \prime}(x),
$$

where $A^{*}$ is defined by equation (2.1). Hence, the governing equations (3.3), (3.4) and (3.5) may be rearranged as follows:

$$
\left\{\begin{array}{l}
E\left(I_{1}+I_{2}\right) v^{\prime \prime \prime \prime}(x)-G b \gamma+p=E I_{t o t} v^{\prime \prime \prime \prime}(x)+p=0 \\
A_{1} u_{1}^{\prime \prime}(x)=-A_{2} u_{2}^{\prime \prime}(x), \\
G b \gamma=E A_{1} u_{1}^{\prime \prime}(x)
\end{array}\right.
$$

where $I_{\text {tot }}$ has been defined by equation (2.1) and corresponds to the momentum of inertia of the monolithic beam.

\subsection{Comparison with Newmark's model.}

Remarkably, the first of Euler's equations (3.3) can be related to the Newmark's forth-order differential equation (2.2), as shown in the sequel.

In the previous section, we have shown that the axial resultant force $N(x)=N_{1}(x)+N_{2}(x)$ is null. Observe now that the bending moment at $x$ in the $i^{\text {th }}$ glass layer, $i=1,2$, is $M_{i}(x)=E I_{i} v^{\prime \prime}(x)$. Consequently, if $N_{1}(x)=-N_{2}(x)$ the resulting bending moment in the whole cross-section of the composite beam is $M(x)=M_{1}(x)+M_{2}(x)+N_{2}(x) H=$ $M_{1}(x)+M_{2}(x)-N_{1}(x) H$, that is

$$
M(x)=E\left(I_{1}+I_{2}\right) v^{\prime \prime}(x)+E A u_{2}^{\prime}(x) H=E\left(I_{1}+I_{2}\right) v^{\prime \prime}(x)-E A u_{1}^{\prime}(x) H .
$$

From this, one finds

$$
\left\{\begin{array}{l}
H A_{1} u_{1}^{\prime}(x)=\left(I_{1}+I_{2}\right) v^{\prime \prime}(x)-M(x) / E, \\
H A_{2} u_{2}^{\prime}(x)=-\left(I_{1}+I_{2}\right) v^{\prime \prime}(x)+M(x) / E .
\end{array}\right.
$$


By substituting (3.19), equation (3.3) can be rewritten as follows:

$$
E\left(I_{1}+I_{2}\right) v^{\prime \prime \prime \prime}(x)-\frac{G b}{t} \frac{A_{1}+A_{2}}{A_{1} A_{2}} I_{t o t} v^{\prime \prime}(x)+\frac{G b}{t E} \frac{A_{1}+A_{2}}{A_{1} A_{2}} M(x)+p=0 .
$$

After setting, as in (2.3) and (2.1), $K:=G b / t$ and $A^{*}=A_{1} A_{2} /\left(A_{1}+A_{2}\right)$, equation (3.20) can be rearranged in the form

$$
v^{\prime \prime \prime \prime}(x)-\frac{K}{E A^{*}} \frac{I_{\text {tot }}}{I_{1}+I_{2}} v^{\prime \prime}(x)+\frac{K}{E^{2} A^{*}} \frac{I_{\text {tot }}}{I_{1}+I_{2}} M(x)+\frac{p(x)}{E\left(I_{1}+I_{2}\right)}=0,
$$

which represents Newmark's equation (2.2).

\section{Approximate simple models}

\subsection{Generalized Newmark (GN) approach.}

Newmark's model represents an accurate elegant re-formulation of the governing differential equations for the composite beam, based upon the association through equilibrium consideration of relevant terms with the bending-moment resultant. However, it presents two major drawbacks. On the one hand, the function $M(x)$ must be known a priori, which is, in general, possible only if the structure is statically determined. On the other hand, the definition of an effective thickness as done in (2.12) or (2.13) is not as straightforward as in Wölfel's approach. Whenever the static constraints render the beam statically undetermined, an approximate solution can be found by considering for $M(x)$ in (3.20) the expression corresponding to the bending moment in a beam of constant thickness under the same static scheme, i.e., with the same constraints of the considered problem. Observe that under this hypothesis $M(x)$ can be easily calculated and does not depend upon the (supposed constant) thickness of the beam. This procedure, as will be shown in section 5, gives in general accurate solutions even if, rigorously speaking, the coupling effect offered by the shear stiffness of the interlayer is variable along the length of the beam; consequently, the effective form of $M(x)$ would coincide with that for a hyperstatic beam with variable cross-sectional height, but cannot be determined a-priori because the cross sectional hight would depend upon $M(x)$ itself.

Once $M(x)$ is given, the fields $v(x) u_{1}(x)$ and $u_{2}(x)$ can be determined by integrating the differential system (3.21), (3.4) and (3.5), with boundary conditions (3.8). The system can be easily solved numerically. Equations (3.18) and (3.19) allow then to determine the bending moment and the axial force in the two glass layers and, consequently, to calculate the maximum stress $\left|\sigma_{(i)}\right|_{\text {max }} \mid$ in the $i^{\text {th }}$ layer through 


$$
\left|\sigma_{(i)}\right| \max =\max _{x}\left|\frac{N_{i}(x)}{A_{i}} \pm \frac{M_{i}(x)}{I_{i}} \frac{h_{i}}{2}\right|
$$

For any particular considered case, once the system of differential equations has been solved, a deflection-effective thickness can be defined by imposing that the maximum sag of a beam with that constant thickness, under the same same constraint and load conditions, coincides with that for the problem solved according to Newmark's theory. This can be easily done by recall that the maximum sag of a beam of constant thickness $h_{w N}^{*}$ is inversely proportional to the moment of inertia $I^{*}$ of the beam itself; for example, for a simply supported beam the maximum sag is $w_{\max }=\frac{5}{384} \frac{q l^{4}}{E I^{*}}=\frac{5}{384} \frac{q l^{4}}{E \frac{b h_{w N}^{* 3}}{12}}$.

Analogously, one can define the stress-effective thickness as the (constant) thickness $h_{\sigma N}^{*}$ of that beam for which the maximum stress $\sigma_{\max }=\frac{6 M_{\max }}{b h_{N}^{*}}$ is equal to the corresponding stress defined by (4.1). The values of the deflection- or stress-effective thickness depend upon the case at hand and, in general, no simple explicit expressions of the same type of (2.12) and (2.13) can be found for Newmark's theory. However, the effective thicknesses may serve as parameters of comparison for various theories and so will be considered for the particular cases analyzed in section 5 .

\subsection{Enhanced effective-thickness (EET) approach.}

The definition of an "effective thickness" for laminated glass represents an efficient method to solve the most various problems, but since Newmark's approach is not able to provide simple explicit expressions, an attempt is now made to consider enhanced approximated solutions through the choice of appropriate and convenient shape functions for the unknown fields $v(x), u_{1}(x)$ and $u_{2}(x)$, whose explicit expression will be found through energetic minimization.

In order to achieve a sufficient approximation, the shape functions for the horizontal and vertical displacements must be compatible with the qualitative properties of the solution. In particular, the proposed approximation must converge to the monolithic limit when $G \rightarrow \infty$, and to the layered limit when $G \rightarrow 0$. Such limits correspond to the solutions of equations (4.2) and (4.3), respectively

$$
\begin{gathered}
E I_{t o t} v^{\prime \prime \prime \prime}(x)+p(x)=0 \quad \Rightarrow v(x)=v_{M}(x) \equiv-\frac{g(x)}{E I_{\text {tot }}}, \\
E\left(I_{1}+I_{2}\right) v^{\prime \prime \prime \prime \prime}(x)+p(x)=0 \quad \Rightarrow v(x)=v_{L}(x) \equiv-\frac{g(x)}{E\left(I_{1}+I_{2}\right)} .
\end{gathered}
$$

Henceforth, the general solution can be sought of the form 


$$
v(x)=-\frac{g(x)}{E I_{R}},
$$

where $I_{R}$ is the equivalent (reduced) moment of inertia defined by

$$
\frac{1}{I_{R}}=\frac{\eta}{I_{t o t}}+\frac{1-\eta}{I_{1}+I_{2}}
$$

Here, the parameter $\eta$ is a non-dimensional quantity, tuning the behavior from the layered limit $(\eta=0)$ to the monolithic limit $(\eta=1)$, while $g(x)$ is the assumed shape function for the vertical displacement, that in general must be selected according to the form of the external load $p(x)$ and the geometric boundary conditions.

Requiring in (3.1) that $\gamma=0$ for the monolithic borderline case, recalling condition (3.6), the horizontal displacements $u_{1}(x)$ and $u_{2}(x)$ can be chosen of the form

$$
u_{1}(x)=\beta \frac{1}{E I_{t o t}} \frac{A_{2}}{A_{1}+A_{2}} H g^{\prime}(x), u_{2}(x)=-\beta \frac{1}{E I_{t o t}} \frac{A_{1}}{A_{1}+A_{2}} H g^{\prime}(x),
$$

where $\beta$ is another non-dimensional parameter, again tuning the response from the layered limit ( $\beta=0$, leading to null horizontal force in the glass layers) to the monolithic limit $(\beta=1$, leading to $\gamma=0)$.

The corresponding energy (3.2) can thus be re-written as a function of the parameters $\eta$ and $\beta$ to give

$$
\begin{aligned}
& \mathfrak{E}\left[u_{1}(x), u_{2}(x), v(x)\right]=\widehat{\mathfrak{E}}[\eta, \beta, g(x)]= \\
& \quad \int_{-l / 2}^{l / 2}\left\{\frac { 1 } { 2 } \left[\frac{\left(I_{1}+I_{2}\right)}{E}\left[\frac{\eta}{I_{t o t}}+\frac{1-\eta}{I_{1}+I_{2}}\right]^{2} g^{\prime \prime}(x)^{2}+\frac{\beta^{2}}{E I_{t o t}^{2}} \frac{A_{1} A_{2}}{A_{1}+A_{2}} H^{2} g^{\prime \prime}(x)^{2}+\right.\right. \\
& \left.\left.+\frac{G b}{t} \frac{H^{2}}{E^{2} I_{t o t}}\left[\frac{\beta}{I_{t o t}}-\frac{\eta}{I_{t o t}}-\frac{1-\eta}{I_{1}+I_{2}}\right]^{2} g^{\prime}(x)^{2}\right]-\frac{p(x)}{E^{2}}\left[\frac{\eta}{I_{t o t}}+\frac{1-\eta}{I_{1}+I_{2}}\right] g(x)\right\} d x,
\end{aligned}
$$

in which the shape function $g(x)$ is a priori given.

Consequently, the minimization of the energy is associated with the minimization with respect to the only free parameters $\eta$ and $\beta$, leading to 


$$
\left\{\begin{array}{l}
\frac{\beta}{I_{t o t}} \frac{A_{1} A_{2}}{A_{1}+A_{2}} \int_{-l / 2}^{l / 2} g^{\prime \prime}(x)^{2} d x+\frac{G b}{t E}\left[\frac{\beta}{I_{t o t}}-\frac{\eta}{I_{t o t}}-\frac{1-\eta}{I_{1}+I_{2}}\right] \int_{-l / 2}^{l / 2} g^{\prime}(x)^{2} d x=0, \\
\left(I_{1}+I_{2}\right)\left[\frac{\eta}{I_{t o t}}+\frac{1-\eta}{I_{1}+I_{2}}\right] \int_{-l / 2}^{l / 2} g^{\prime \prime}(x)^{2} d x+ \\
\quad+\frac{G b}{t E} H^{2}\left[\frac{\beta}{I_{t o t}}-\frac{\eta}{I_{t o t}}-\frac{1-\eta}{I_{1}+I_{2}}\right] \int_{-l / 2}^{l / 2} g^{\prime}(x)^{2} d x-\int_{-l / 2}^{l / 2} p(x) g(x) d x=0 .
\end{array}\right.
$$

The system (4.8) can be substantially simplified provided that a particular shape function $g(x)$ is chosen. To illustrate, suppose that $g(x)$ is such that $v(x)=-\frac{g(x)}{E I_{R}}$ represent the solution of the elastic bending of a beam with constant moment of inertia $I_{R}$ under the load $p(x)$, with the same boundary condition of the problem at hand. Consider the virtual work equality for this system in which the aforementioned $v(x)$ is selected as the strain/displacement field, whereas the bending moment in equilibrium with $p(x)$ is given by $M(x)=v^{\prime \prime}(x) E I_{R}$. The external and internal virtual work can be written as

$$
L_{v e}=\int_{-l / 2}^{l / 2} p(x) \frac{g(x)}{E I_{R}} d x, \quad L_{v i}=\int_{-l / 2}^{l / 2} M(x) v^{\prime \prime}(x) d x=\int_{-l / 2}^{l / 2} g^{\prime \prime}(x) \frac{g^{\prime \prime}(x)}{E I_{R}} d x
$$

so that the equality between external and internal virtual work leads to

$$
\int_{-l / 2}^{l / 2} p(x) g(x) d x=\int_{-l / 2}^{l / 2} g^{\prime \prime}(x)^{2} d x
$$

This condition can be used to simplify (4.8), yielding the following noteworthy expression for $\beta$ and $\eta$ that in this particular case coincide, i.e.,

$$
\eta=\beta=\frac{1}{1+\frac{I_{1}+I_{2}}{\mu I_{t o t}} \frac{A_{1} A_{2}}{A_{1}+A_{2}} \frac{\int_{-l / 2}^{l / 2} g^{\prime \prime}(x)^{2} d x}{\int_{-l / 2}^{l / 2} g^{\prime}(x)^{2} d x}}
$$

where the nondimensional coefficient $\mu$, defined through

$$
\mu=\frac{G b}{E t},
$$

represents a measure of the elastic stiffness of glass with respect to the stiffness of the interlayer. 
It should also be noticed that the coefficient $\eta$ (or $\beta$ ) that appears in the definition of $I_{R}$ as per (4.5), is somehow similar to the parameter $\Gamma$ of (2.6). In fact, the layered limit corresponds to $\Gamma=\eta=0$ and the monolithic limit to $\Gamma=\eta=1$, but comparing (4.5) and (2.6) one can realize that the values of both parameters coincide only on the borderline cases. More precisely, $\Gamma$ and $\eta$ are associated with the weighted arithmetic and geometric mean of $I_{\text {tot }}$ and $I_{1}+I_{2}$, respectively. In any case, analogously to the expression (2.12) for $\Gamma$, also the parameter $\eta$ can be used to define the deflection-effective thickness $\hat{h}_{e f ; w}$ that, from (4.5), turns out to be

$$
\hat{h}_{w}=\sqrt[3]{\frac{1}{\frac{\eta}{\left(h_{1}^{3}+h_{2}^{3}+12 I_{s}\right)}+\frac{(1-\eta)}{\left(h_{1}^{3}+h_{2}^{3}\right)}}}
$$

$I_{s}$ having been defined in (2.10).

For the calculation of the maximum (in absolute value) bending stress $\left|\sigma_{(i)}\right|_{\max }$ in the $i$-th glass layer, $i=1,2$, it is possible to use the stress-effective thickness $\hat{h}_{i ; e f ; \sigma}$, analogous to that defined in (2.13) for Wölfel's model, defined through

$$
\left|\sigma_{(i)}\right| \max =\frac{\max _{x}|M(x)|}{\frac{1}{6} b \hat{h}_{i ; e f ; \sigma}^{2}}=\max _{x}\left|\frac{N_{i}(x)}{A_{i}} \pm \frac{M_{i}(x)}{I_{i}} \frac{h_{i}}{2}\right|,
$$

where, recalling (4.4), (4.6) and (4.11),

$$
N_{i}(x)=E A_{i} u_{i}^{\prime}(x)=-(-1)^{i} \frac{\eta}{I_{\text {tot }}} A^{*} H g^{\prime \prime}(x), M_{i}(x)=E I_{i} v^{\prime \prime}(x)=-\frac{I_{i}}{I_{R}} g^{\prime \prime}(x)
$$

Recalling the definitions of $h_{s ; 1}$ and $h_{s ; 2}$ of (2.10), one finds from (4.14) the following expression for the stress-effective thickness:

$$
\hat{h}_{1 ; \sigma}=\sqrt{\frac{1}{\frac{2 \eta h_{s ; 2}}{h_{1}^{3}+h_{2}^{3}+12 I_{s}}+\frac{h_{1}}{\hat{h}_{e f ; w}^{3}}}}, \quad \hat{h}_{2 ; \sigma}=\sqrt{\frac{1}{\frac{2 \eta h_{s ; 1}}{h_{1}^{3}+h_{2}^{3}+12 I_{s}}+\frac{h_{2}}{\hat{h}_{e f ; w}^{3}}}} .
$$

Clearly, the expressions for the equivalent thickness for deflection and stress are different from those of (2.12) and (2.13), defined by Bennison et al. [18]. A detailed comparison will be made in the following section. 


\section{$5 \quad$ Examples}

The results obtainable with the approximate approaches of sections 4.1 and 4.2 are now compared with those proposed by Bennison et al. [17], that relies upon Wölfel's theory of section 2, and with the numerical solutions of a FEM model, which considers the effective stiffness of the interlayer in the composite package. Numerical simulations have been made with the program Abaqus, using a 3-D mesh with solid 20-node quadratic bricks with reduced integration, available in the program library [20].

By imposing that the maximum sag evaluated through the finite element analysis of a beam coincides with that one with that constant thickness, under the same same constraint and load conditions, the deflection-effective thickness may be easily evaluated. Furthermore, the F.E. analysis allows to calculate the maximum stress in the beam, that, since the maximum bending moment is known, allows to evaluate the stress-effective thickness.

The four paradigmatic cases represented in fig. 4 have been considered for the sake of comparison because they represent beams under different boundary and loading conditions. Assumed structural parameters for all cases are $l=3150 \mathrm{~mm}, b=1000 \mathrm{~mm}, h_{1}=h_{2}=$ $10 \mathrm{~mm}, t=0.76 \mathrm{~mm}, E=70 G P a$, while the modulus $G$ of the polymeric interlayer is varied to evaluate its influence on the shear-coupling of the glass plies. The distributed pressure on the beam is taken equal to $0,75 \mathrm{KN} / \mathrm{m}^{2}$ so that, with $b=1000 \mathrm{~mm}$, the distributed load per unit length becomes $p=0,75 \mathrm{~N} / \mathrm{mm}$. For the concentrated force in fig. $4 b$, we take $F=1 k N$.

a)

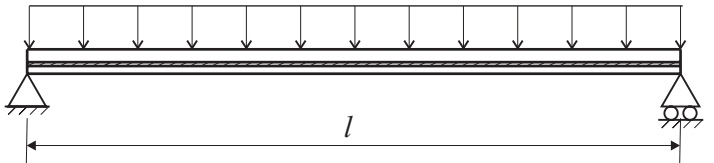

b)

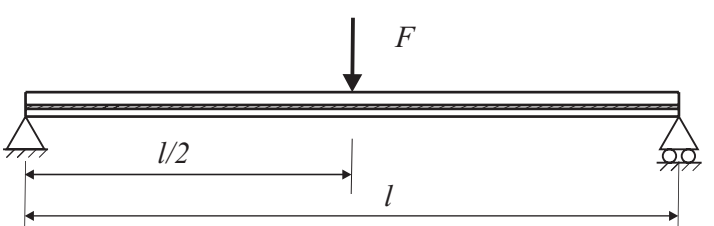

c)

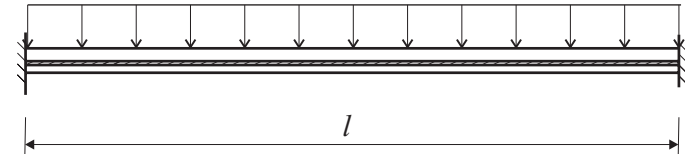

d)

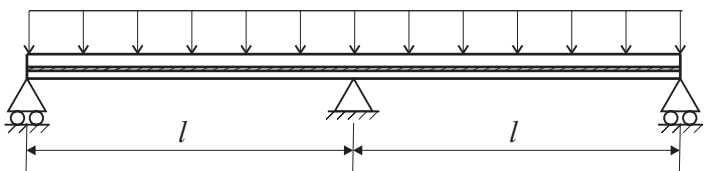

Figure 4: Representative examples of laminated glass beams under different boundary and load conditions.

The mesh employed in the numerical simulations can be recognized in fig. 5, which shows the stress state for the double-clamped beam of fig. $4 c$. The fine meshing allows to accurately determine the stress and strain fields.

It should be mentioned that the numerical model of fig. 5 corresponds to a plate rather than a beam and, consequently, there may be a difference with the 1-D beam theory for what the deflection is concerned. When the width of the plate is comparable with its length, 


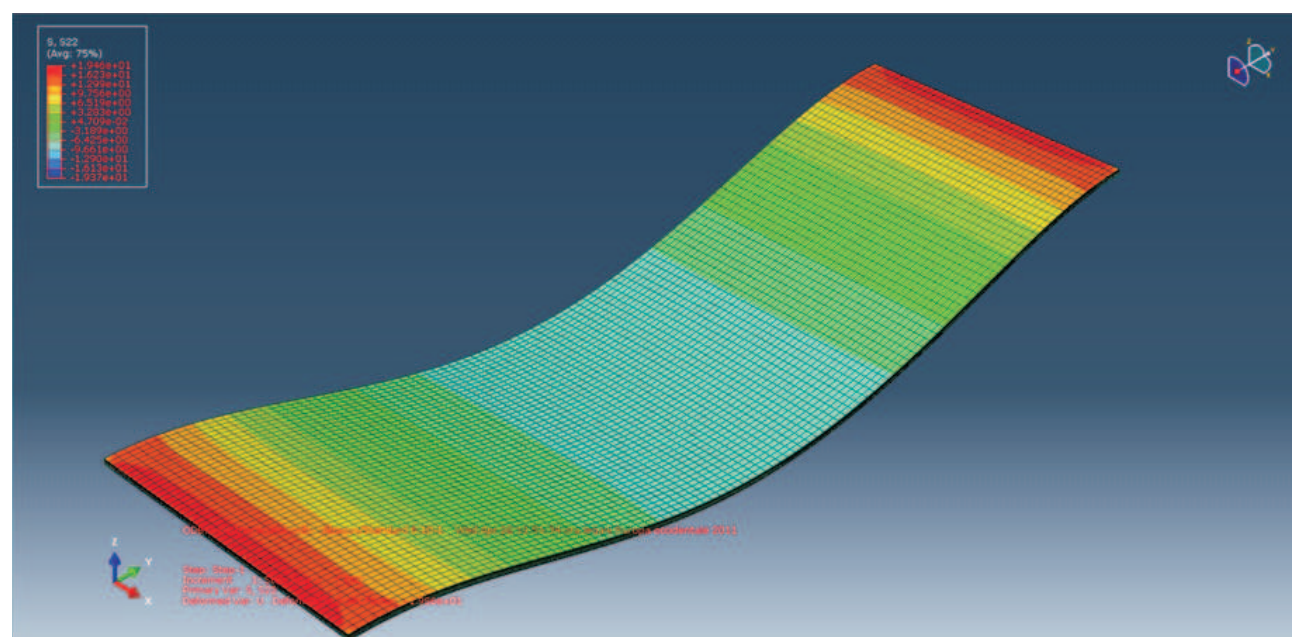

Figure 5: Axial stress of a double-clamped beam under uniformly distributed load, plotted on the deformed shape.

this can be up to a multiplier of the order of $1-\nu^{2}$, where $\nu$ is the Poisson's ratio of glass, i.e., $\nu=0.2$. Here, the length of the plate is about 3.5 times its width and consequently the difference with beam theory is expected to be considerably smaller than that. In any case, since the effective thickness approach is used to calculate plates of laminated glass, here the comparison has been made on purpose with a geometry that can be found in the design practice. The differences with the ideal case $\nu=0$ are found to be less than $2 \%$.

\subsection{Simply supported beam under uniformly distributed load.}

For the case of the simply supported beam shown in figure $4 a$, the boundary conditions (3.8) give $v( \pm l / 2)=0, v^{\prime \prime}( \pm l / 2)=0, u_{1}^{\prime}( \pm l / 2)=u_{2}^{\prime}( \pm l / 2)=0$. Consider as a shape function in (4.4) the function $g(x)$ associated with the elastic deflection of a monolithic simply supported beam under uniformly distributed load $p$, i.e.,

$$
g(x)=-p\left(\frac{1}{24} x^{4}-\frac{1}{16} l^{2} x^{2}+\frac{5}{384} l^{4}\right) .
$$

Such choice, through (4.4) and (4.6), leads to expressions for $v(x), u_{1}(x)$ and $u_{2}(x)$ that satisfy the aforementioned boundary conditions. Moreover, it is compatible with (4.10), so that (4.11) holds and $\eta=\beta$.

By substituting the shape function (5.1) into equation (4.11), the following expression for coefficient $\eta=\beta$ may be obtained: 


$$
\eta=\beta=\frac{1}{1+\frac{168}{17 \mu l^{2}} \frac{I_{1}+I_{2}}{I_{\text {tot }}} \frac{A_{1} A_{2}}{A_{1}+A_{2}}} .
$$

For a shear modulus of the polymeric interlayer $G$ varying from $0,01 M P a$ to $10 M P a$, the graphs of figure 6 compare the deflection- and stress-effective thickness, calculated according to the proposed enhanced effective thickness (EET) approach of section 4.2, as per equations (4.16) and (4.13), with the effective thickness according to Wölfel-Bennison (WB) of (2.13) and (2.12), as well as with the effective thickness evaluated by adopting the revised generalized Newmark (GN) model of sec. 4.1, that for this statically determinate case coincides with the classical Newmark formulation of [2]. Observe that here all the models give results that in practice coincide, a finding that is not surprising because this is the simplest case upon which all the approaches have been calibrated. The good approximation achieved by the simplified approaches is evidenced by a comparison with the numerical results, also reported in the same figure.

a)

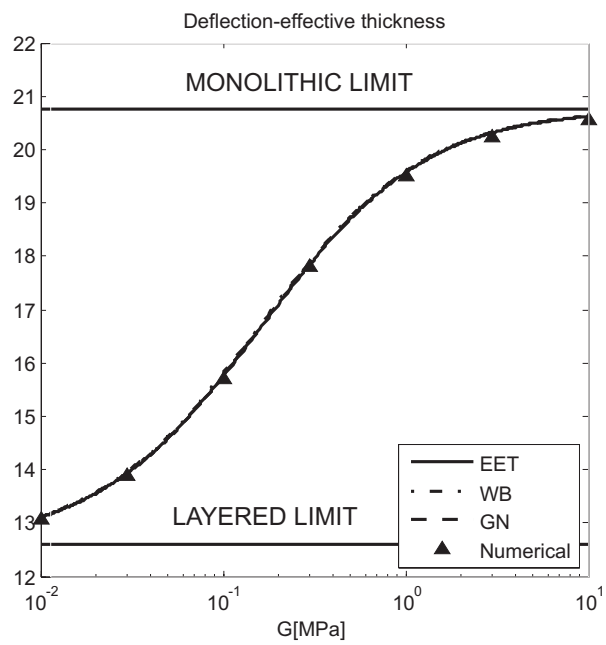

b)

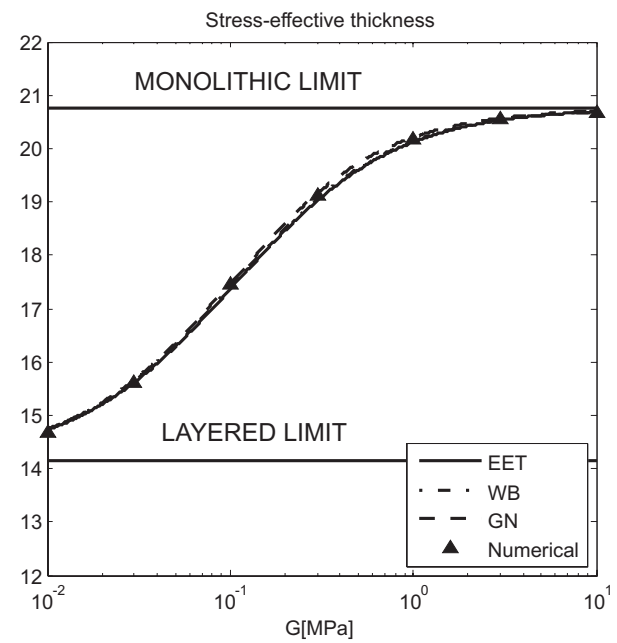

Figure 6: Simply supported beam under uniform load. Comparison of the effective thicknesses obtained with: Wölfel-Bennison approach (WB); generalized Newmark's approach (GN); enhanced effective-thickness approach (EET). Results from the numerical experiments are also reported for the sake of comparison.

Fig. 7 shows the values of the maximum sag of the beam as a function of the interlayer shear modulus $G$. Again, the numerical output confirms the accuracy of all the simplified approaches. 


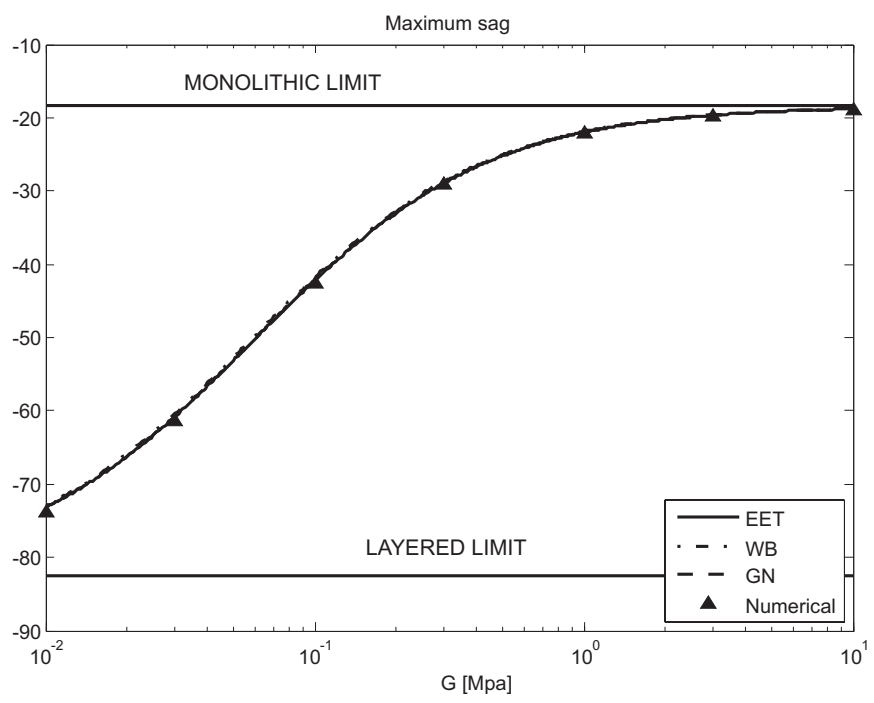

Figure 7: Simply supported beam under uniform load. Comparison of the maximum sag obtained with the proposed simplified approaches, generalized Newmark (GN) and enhanced effective thickness (EET), and with the model by Wölfel-Bennison (WB). The results from numerical experiments are also indicated.

\subsection{Simply supported beam under concentrated load.}

Consider the same beam, but now under a concentrate load $F$ at midspan (fig. $4 b$ ). The boundary conditions are the same as before and the function $g(x)$ to be considered in (4.4) is now

$$
g(x)=\left\{\begin{array}{l}
-F\left(-\frac{1}{12} x^{3}-\frac{1}{8} l x^{2}+\frac{1}{48} l^{3}\right) \quad \text { for } \quad-l / 2 \leq x \leq 0, \\
-F\left(\frac{1}{12} x^{3}-\frac{1}{8} l x^{2}+\frac{1}{48} l^{3}\right) \quad \text { for } \quad 0<x \leq l / 2 .
\end{array}\right.
$$

Thus, after substitution into (4.11), one finds

$$
\eta=\beta=\frac{1}{1+\frac{10}{\mu l^{2}} \frac{I_{1}+I_{2}}{I_{\text {tot }}} \frac{A_{1} A_{2}}{A_{1}+A_{2}}} .
$$

Figure 8 shows, as a function of $G$, comparisons of the enhanced effective thicknesses (EET) calculated with equations (4.16) and (4.13), with the effective thicknesses calculated through expressions (2.13) and (2.12) for the Wölfel-Bennison (WB) model [18]. Notice that, also for this load condition, both approaches give results that practically coincide. As a matter of fact, it was observed in sec. 2 that Wölfel's theory prescribes for this case a coefficient $\beta=12$ in (2.4), instead of 9.6 as indicated by the formulation of Wölfel-Bennison 
a)

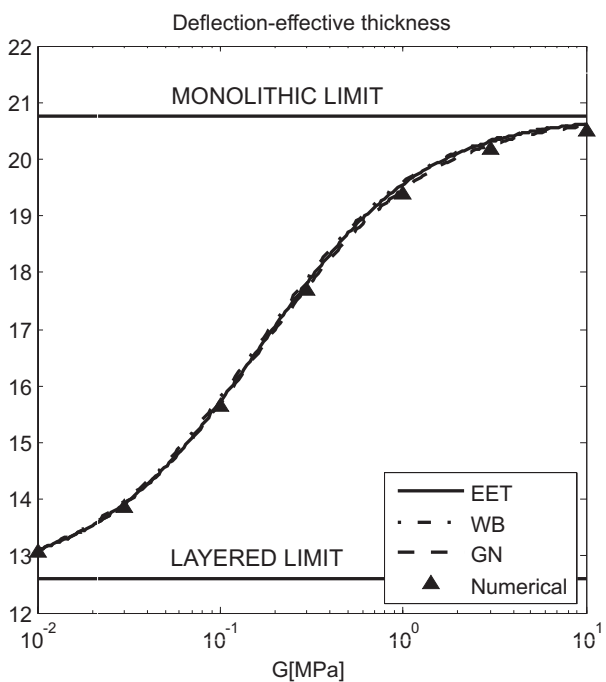

b)

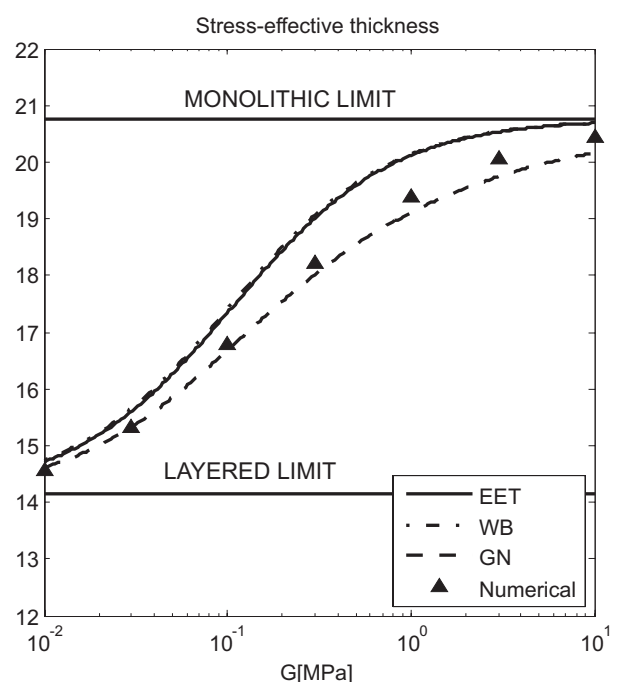

Figure 8: Simply supported beam under concentrated load. Comparison of the effective thicknesses obtained with: Wölfel-Bennison (WB) approach; the generalized Newmark model (GN); the enhanced effective thickness (EET) approach; the numerical simulations.

[18]. However, at least for the case at hand, the difference is not substantial and the values of the effective thicknesses that would be obtained using either 12 or 9.6 are almost the same, at least when $G$ varies in the aforementioned range.

Further comparing these results with those obtainable with the revised generalized Newmark (GN) model (coinciding also in this case with the classical formulation [2]), it is evident from figure 8 that the deflection-effective thickness coincides with those of both aforementioned formulations, whereas the stress-effective thickness is qualitatively different, especially in those branches tending to the monolithic limit. The numerical simulations, whose results are also reported in the same figure, are in good agreement with Newmark theory. This is not surprising because, as already noticed in sec. 4.1, Newmark theory is very accurate when the diagram of bending moment is a priori known, as it is the case in a statically determined structure.

The maximum deflexion of the beam, calculated with the three formulations, is recorded as a function of $G$ in Figure 9. One may observe that the enhanced effective-thickness method predicts a slightly-stiffer beam than Wölfel-Bennison formulation, but the difference is very very small. The numerical experiments, also reported in the same figure, confirm the prediction of the approximate models. 


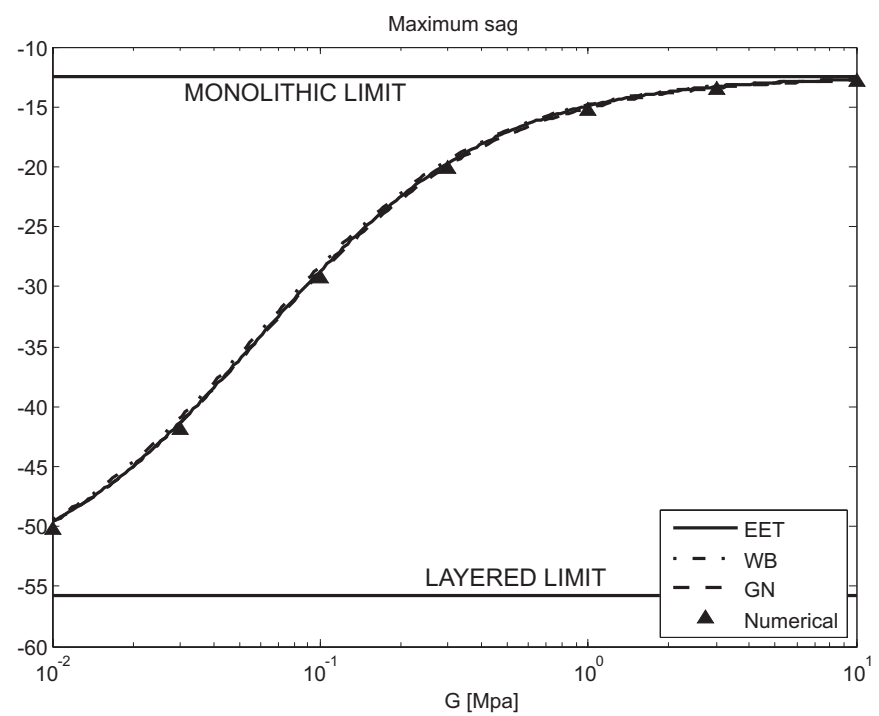

Figure 9: Simply supported beam under concentrated load. Maximum sag obtained with: Wölfel-Bennison (WB) approach; the revised Newmark (GN) model; the enhanced effective thickness (EET) approach; the numerical simulations.

\subsection{Double clamped beam under uniform load.}

What distinguishes the case of the double clamped beam (fig. 4c) from the previous ones is that now the structure is statically undetermined. The boundary conditions (3.8) for $v(x)$ are of the form $\left.v( \pm l / 2)=0, v^{\prime}( \pm l / 2)=\right)$, while for what the horizontal displacement is concerned, we set $u_{1}^{\prime}( \pm l / 2)=u_{2}^{\prime}( \pm l / 2)=0$. These conditions imply that the beam ends cannot rotate but the border is stress free. As the shape function in (4.4) consider then the function $g(x)$ defined as

$$
g(x)=-p\left(\frac{1}{24} x^{4}-\frac{1}{48} l^{2} x^{2}+\frac{1}{384} l^{4}\right),
$$

associated with the elastic curve of a monolithic beam under the same load. This choice is compatible with (4.10) and leads in (4.4) and (4.6) to expressions for $v(x), u_{1}(x)$ and $u_{2}(x)$ that respect all the boundary conditions (3.8). Then, substituting into (4.11), the non-dimensional coefficient $\eta$ turns out to be (also for this case $\eta=\beta$ )

$$
\eta=\beta=\frac{1}{1+\frac{42}{\mu l^{2}} \frac{I_{1}+I_{2}}{I_{\text {tot }}} \frac{A_{1} A_{2}}{A_{1}+A_{2}}} .
$$

Figure 10 shows in particular the comparison of the enhanced effective thicknesses (EET), calculated through equations (4.16) and (4.13), with the Wölfel-Bennison (WB) 

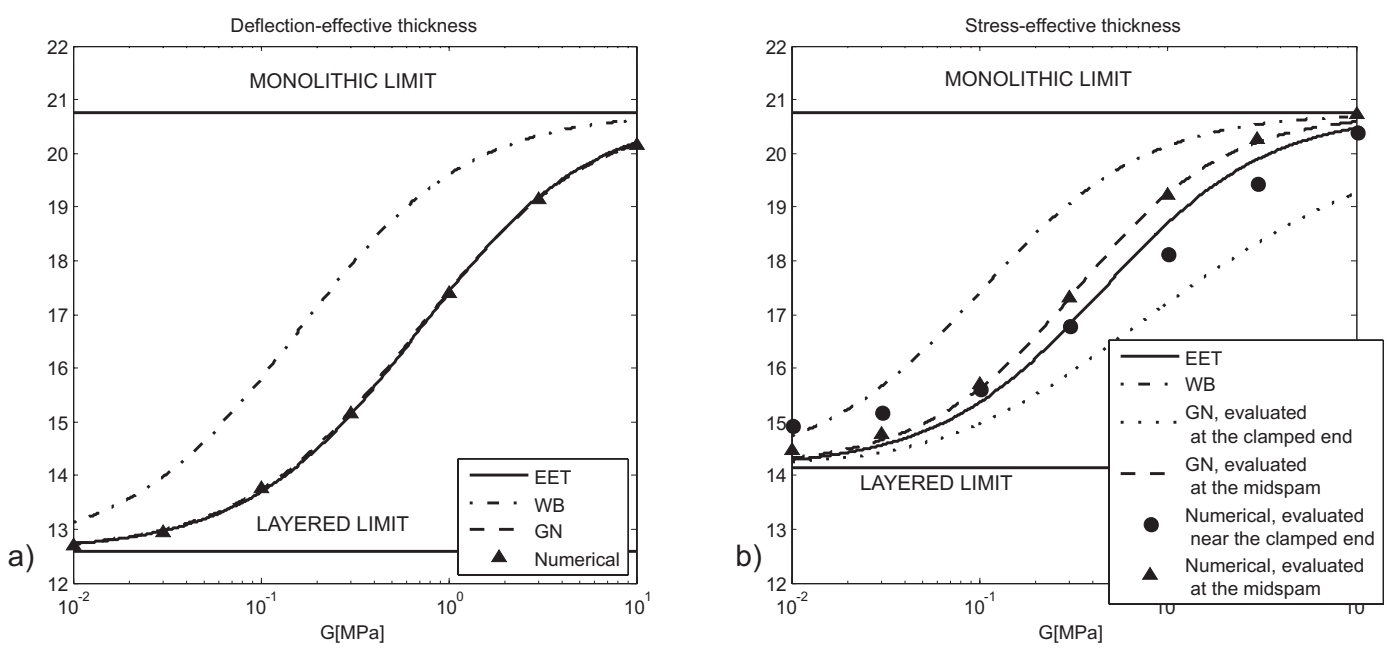

Figure 10: Double clamped beam under uniform load. Comparison of the effective thicknesses obtained with: Wölfel-Bennison (WB) approach; the generalized Newmark (GN) model (at two cross sections); the enhanced effective thickness (EET) approach; the numerical simulations (at two cross sections).

effective thicknesses defined in (2.13) and (2.12) as per [18]. What is evident here is that the proposed EET approach and the WB formulation give different results at the qualitative level, especially for low values of $G$. Most of all, WB is not on the side of safeness, because it predicts deflection and stress values much lower than those predicted by our formulation.

The aforementioned approaches rely upon simplifying assumptions that provide a correspondence between the laminated beam and a monolithic beam of constant thickness. However, if one considered the actual response of the laminated beam through, e.g., an accurate numerical model, one would find that the bonding effect offered by the interlayer varies from section to section. Since the effective thickness depends upon the bonding offered by the interlayer, the correct correspondence would be with a monolithic beam with variable cross section. This correspondence, however, is difficult to determine because the effective cross-sectional inertia depends upon the form of the diagram of the bending moment, but the distribution of bending moments in a hyperstatic beam depends upon the cross-sectional inertia. In any case, once the laminated beam problem has been accurately solved, one may define for each cross section a stress-effective thickness as the thickness of a hypestatic monolithic beam with constant cross section that exhibits, at the section under consideration, the same maximal stress of the laminated beam.

For the sake of comparison, the stress-effective thickness defined as above has been derived from the numerical experiments in two representative cross sections, i.e., in proximity of the clamped edges and at the midspan of the beam. Remarkably, as shown in fig. 10, our EET formulation furnishes an average value of the stress-effective thickness. Notice as well that the formulation WB does not provide accurate results. This is not surprising because, 
as already observed in sec. 2 when the structure is not statically determined Wölfel's hypotheses do not hold. The inaccuracy of the approach WB is also confirmed by the graphs of the maximum deflexion of the beam, recorded in figure 11.

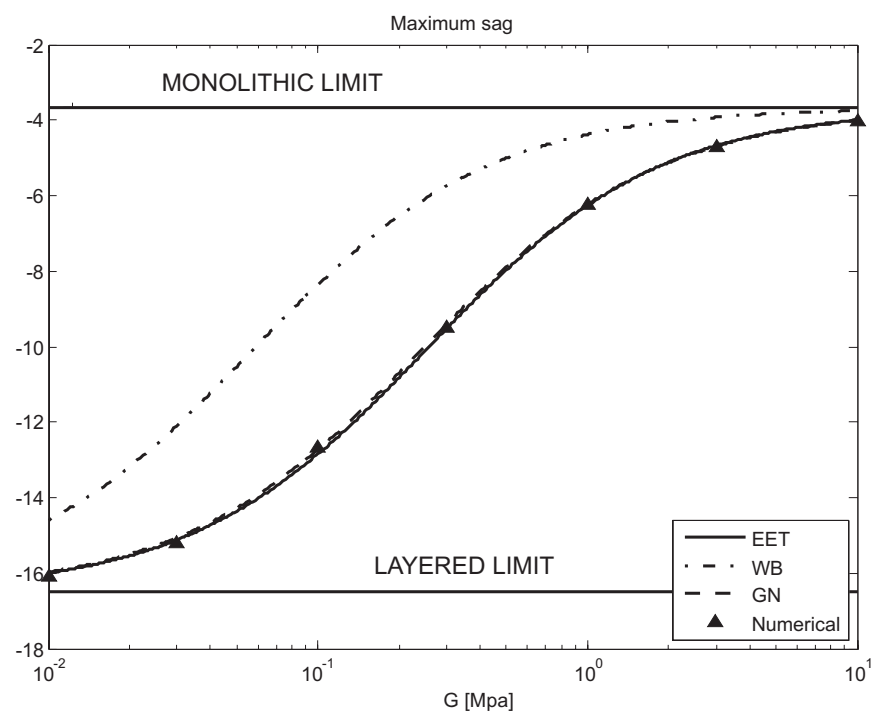

Figure 11: Double clamped beam under uniform load. Maximum sag obtained with: WölfelBennison (WB) approach; the generalized Newmark (GN) model; the enhanced effective thickness (EET) approach; the numerical simulations.

It is interesting for this case to discuss the results obtainable with the revised Newmark model (GN) of sec. 4.1 that, we recall, relies upon the simplifying assumption that $M(x)$ has the same form of the bending moments in a hyperstatic beam with constant thickness. Observing first figs. $10 a$ and 11 , it is clear that GN well captures the deflection of the beam. For what the stress is concerned, this approach can account for the variability of the bonding effect of the interlayer along the beam axis and, consequently, the stress effective thickness is sectional-dependent as mentioned above. It is then evident from fig. $10 b$ that GN is able to accurately provide the stress at the beam midspan, but it is not so precise when stress is evaluated at the clamped ends.

The re-visitation of Newmark's approach may be perhaps better understood from fig. 12 , where the deflection curve is compared with that of the enhanced effective-thickness approach of sec. 4.2 for the case $G=1 M P a$. Indeed, the latter one is determined by the choice (5.5) for the shape function $g(x)$ that, for the case at hand, is derived from the elastic curve of a double clamped beam with uniform cross section. On the other hand, the GN model assumes the distribution of the bending moment of the double clamped beam with uniform cross section but not the corresponding deformation and, indeed its deflection curve differs from that of that of the constant-thickness beam, though the sag at midspan is the same. This is another evidence of how the variability of the bonding stiffness offered by the interlayer in a hyperstatic beam may effect its deformation, that would correspond 


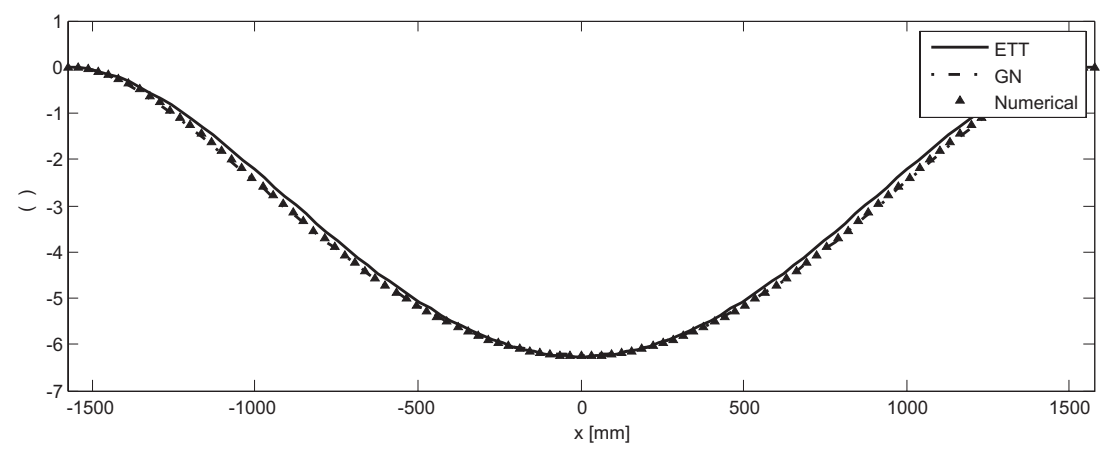

Figure 12: Deformed shape of double clamped beam under uniform load. Comparison of results obtained with the enhanced effective-thickness (EET) approach, the generalized Newmark (GN) approach and the numerical experiments.

to that of a hyperstatic beam with variable cross section. In the same figure the results of the numerical simulation, which are in excellent agreement with the GN predictions.

\subsection{Beam with three supports under uniform load.}

The case of the beam with three supports under uniformly distributed load, shown in fig. $4 d$, represents an intermediate case between those of figs. $4 a$ and $4 c$. In fact, using the symmetry, only half of the system, say $0 \leq x \leq l$, can be considered with boundary conditions in (3.8) of the type $v(0)=v(l)=0, v^{\prime}(0)=0, v^{\prime \prime}(l)=0$. For this case, we set as a shape function in (4.4) the expression

$$
g(x)= \begin{cases}-p\left(\frac{1}{24} x^{4}+\frac{5}{48} l x^{3}+\frac{1}{16} l^{2} x^{2}\right) & \text { for } \quad-l \leq x \leq 0, \\ -p\left(\frac{1}{24} x^{4}-\frac{5}{48} l x^{3}+\frac{1}{16} l^{2} x^{2}\right) & \text { for } \quad 0<x \leq l .\end{cases}
$$

This choice implies through (4.6) that $u_{1}^{\prime}(0)=u_{1}^{\prime}(l / 2)=u_{2}^{\prime}(0)=u_{2}^{\prime}(l / 2)=0$, which is not completely consistent because at the symmetry section $x=0$ one should assume $u_{1}(0)=u_{2}(0)=0$. In fact, in general, $u_{1}^{\prime}(0) \neq 0$ and $u_{2}^{\prime}(0) \neq 0$ because the resultant axial force in each glass ply is not necessarily null at the symmetry section. However, we will verify later on that this approximation does not considerably affect the results.

Again, the assumed shape functions corresponds to the deflection curve of the corresponding statically indeterminate problem, implying $\eta=\beta$. Substituting in the relevant expressions, one thus obtains

$$
\eta=\beta=\frac{1}{1+\frac{21}{\mu l^{2}} \frac{I_{1}+I_{2}}{I_{\text {tot }}} \frac{A_{1} A_{2}}{A_{1}+A_{2}}} .
$$


The comparison between the deflection-effective thicknesses calculated with the three approaches is represented in figure $13 a$ together with numerical experiments. Here, we can notice a substantial deviation of Wölfel-Bennison approach especially for the lowest values of $G$, but the numerical experiments are in favor of the enhanced effective-thickness approach, which gives results that in practice coincide with the revised Newmark (GN) theory. This finding is confirmed by the graphs of the maximum deflexion of the beam, recorded in figure 14.

a)

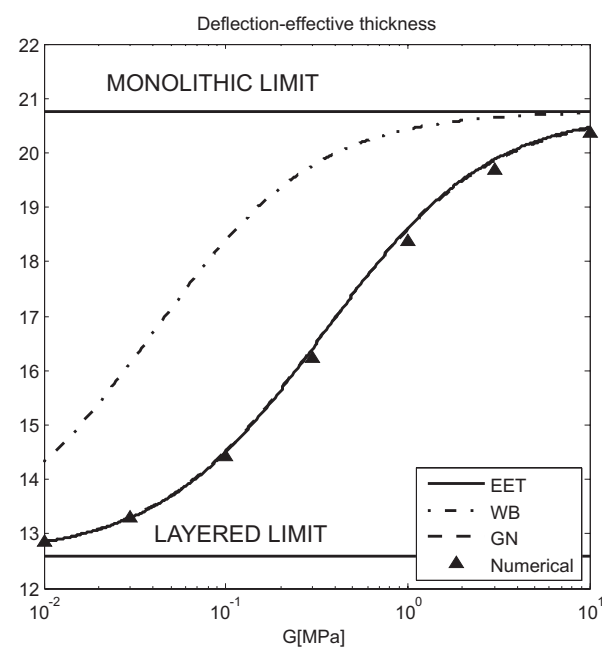

b)

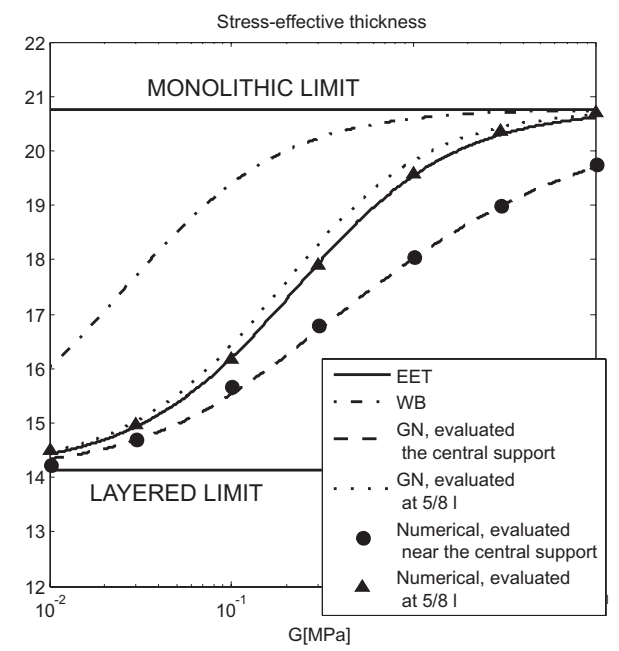

Figure 13: Beam with three supports under uniform load. Comparison of the effective thicknesses obtained with: Wölfel Bennison (WB) approach; the generalized Newmark (GN) model (at two representative cross sections); the enhanced effective thickness (EET) approach; the numerical simulations (at two cross sections).

For what the stress-effective thickness is concerned, since the system is not statically determined, from the results of the numerical simulations, as well as from the GN approach, we have considered two representative cross sections, one in the neighborhood of the central support $x=0$, the other one at $x=5 l / 8$, where the bending moment should be extremal. For this case, the enhanced effective-thickness (EET) approach gives results that well capture the stress in the middle of the beam, but are not so accurate at the symmetry section. On the other hand, the revised Newmark (GN) model gives excellent results at both cross sections.

The results that are obtained with the enhanced effective-thickness (EET) approach are summarized, for the sake of comparison, in fig. 15 that shows the deflection of the beam in the four configurations of fig. 4 for different values of $G$. The bonding performance of the interlayer is clearly recognizable by the comparison with the monolithic and layered limits. Even small values of $G$ (of the order of $1 M P a$ ) are sufficient to provide a considerable bonding strength that is sufficient to produce a response remarkably close to that of the monolithic limit. This is why an accurate definition of the effective thickness, especially in 


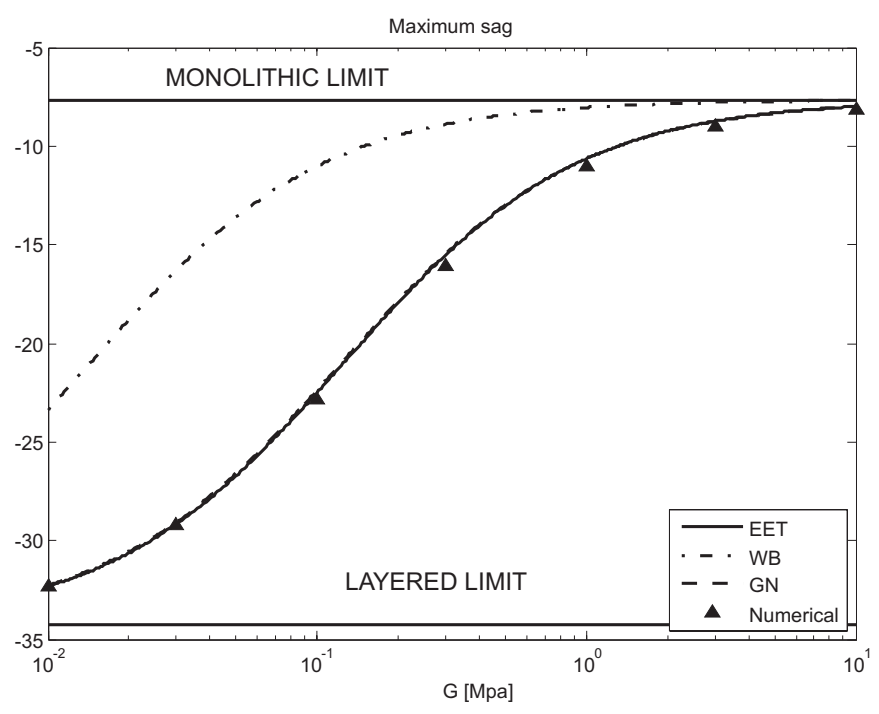

Figure 14: Beam with three supports under uniform load. Maximum sag obtained with: Wölfel-Bennison (WB) approach; the generalized Newmark (GN) model; the enhanced effective thickness (EET) approach; the numerical simulations.

the case of soft interlayers, is crucial to achieve a good design on the side of slenderness, lightness and economy.

\section{Discussion and Conclusions}

The two major simple approaches to the structural design of laminated glass are essentially Newmark model [2] and the approach by Bennison et al. [17] [18], which is based upon the original work by Wölfel [4]. These two formulations have been reconsidered here and a common denominator to them, traditionally reputed completely different, has been found by considering the problem within a variational framework. In particular, it has been shown that the model by Wölfel-Bennison derives from assuming a proper shape function for the beam deformation in the corresponding strain energy functional, whereas the approach by Newmark recognizes that some relevant terms in the governing Euler-Lagrange equations represent the resultant bending moment at the composite cross section. Roughly speaking, Wölfel-Bennisonl is based upon an assumption for the deformation field, whereas in Newmark it is the bending moment distribution that must be known a priori. More in particular, Wölfel-Bennison approach assumes for the laminated glass beam a deflection curve similar in type to the elastic curve of simply supported beam under uniformly distributed load and, consequently, turns out to be accurate when the case at hand reflects these hypotheses. On the other hand, Newmark model is perfectly applicable and gives excellent results only when the beam is statically determined, i.e., when the bending moment 
a)

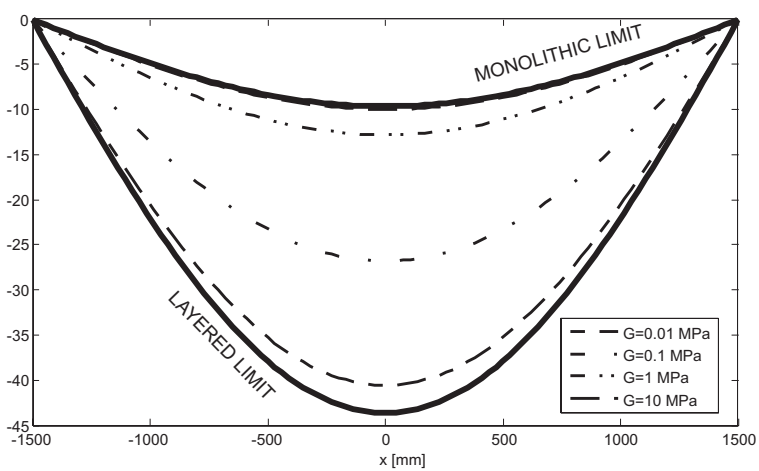

b)

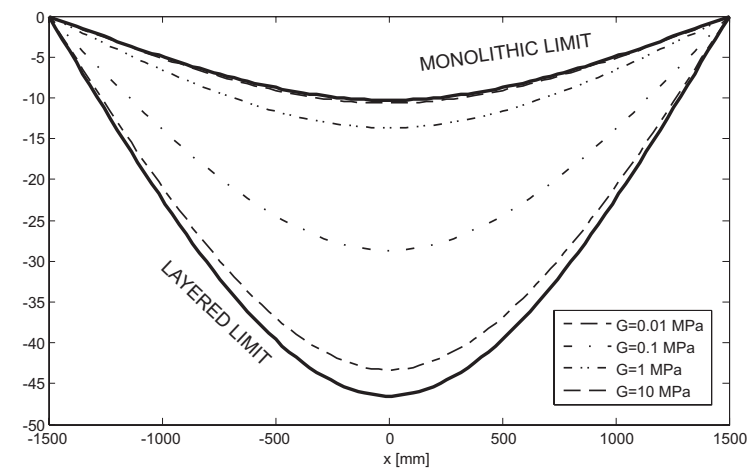

c)

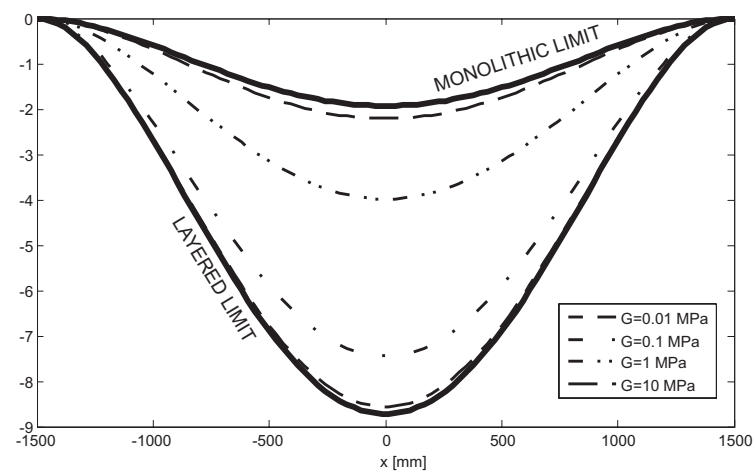

d)

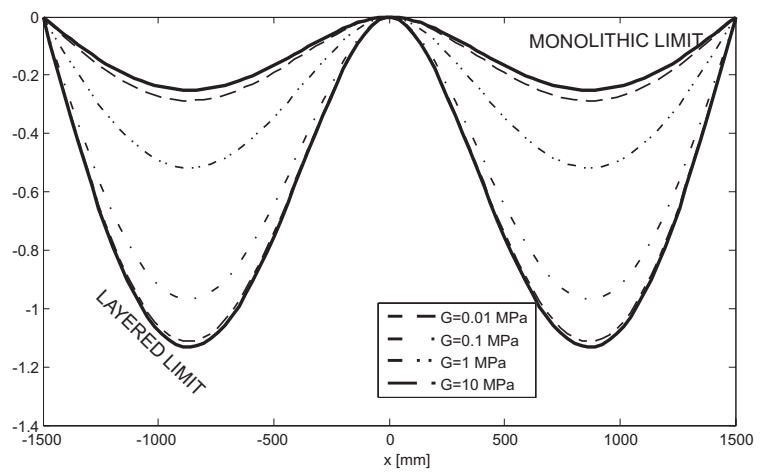

Figure 15: Deformed shape of the laminated glass beam calculated with the enhanced effective-thickness (EET) approach. Case $h 1=h 2=10 \mathrm{~mm}, t=0.76 \mathrm{~mm}, l=3150 \mathrm{~mm}$, $b=10000 \mathrm{~mm}, E=70000 \mathrm{MPa}, p=500 \mathrm{~N} / \mathrm{m} F=1000 \mathrm{~N}$ 
diagram is precisely determinable.

Here, we have revised these two approaches and proposed possible generalizations. For what the Newmark formulation is concerned, in order to deal with hyperstatic structures the proposal is to consider for the bending moment that distribution corresponding to the case of a beam of uniform constant thickness under the same boundary and load conditions of the problem at hand. We have verified with representative examples and comparison with accurate numerical experiments that this position usually provides excellent results, both for the deflection and the stress calculation. On the other side, to extend WölfelBennison calculations we have proposed to assume various shape function for the laminated beam deflection, which should reflect the effective boundary and load conditions of the structure under consideration. This formulation results particularly simple if one consider as the shape function the form of the elastic curve of a monolithic beam with constant cross section under the same conditions. With this position, the proposed approach gives excellent results and relatively compact formulas.

We have also verified that the approach $\grave{a} l a$ Wölfel-Bennison allows to naturally define a deflection- and stress-effective thickness as per equations (2.12) and (2.13), which is very convenient for the structural design. Here, we have extended such formulas to the case of more elaborated boundary and load conditions, reaching simple expressions for the effective thickness, recorded in (4.13) and (4.16), that can be easily adapted to shape functions of any form through the introduction of the coupling parameter $\eta$, defined in (4.11), that varies between the threshold value 0 (layered limit) and 1 (monolithic limit). The calculation of the effective thickness according to the enhanced proposed approach thus presents no additional difficulty with respect to the traditional Wölfel-Bennison formulation, but gives much better results when the beam is not simply supported and the load is not uniform, especially when the interlayer is soft and the laminated beam approaches the layered limit. The enhanced effective-thickness approach here proposed in section 4.2 thus seems to represent a powerful tool for the calculation of laminated glass. In particular, it is much simpler than the models à la Newmark, for which it is necessary to calculate a priori the bending moment in the beam and, most of all, the evaluation of an effective thickness is not so natural and does not lead to compact expression. In particular, the stress-effective thickness depends upon the particular section at which the stress needs to be evaluated.

Last but not least, all the models here considered are valid only for the 1-D scheme of a laminated glass beam under flexure, i.e., when the glass panel is constrained at two borders and the deformation is cylindrical. On the other hand, it is customary in the design practice to use the effective thickness calculated according to Wölfel-Bennison, using the expression (2.12) and (2.13), to estimate the state of stress and the deformation of a laminated plate, under the most various boundary and load conditions. This procedure needs to be questioned since we have demonstrated here that the aforementioned formulation does not give in general accurate results,except for the case of a simply supported 1-D beams. The extension of the effective-thickness notion to the case of plates and shells under bending is the subject of presently ongoing work. 
Acknowledgements. The authors acknowledge the Italian MURST for partial support under the PRIN2008 program.

\section{References}

[1] Love, A.E., A Treatise on the Mathematical Theory of Elasticity, Dover, New York, 1944.

[2] Newmark, N.M., Siess, C.P., Viest, I.M., Test and analysis of composite beams with incomplete interaction, Proceedings of the Society for Experimental Stress Analysis, $\mathbf{9}$, pp. 75-92, 1951.

[3] Hooper, J.A., On the bending of architectural laminated glass, Int. J. of Mech. Sciences, 15, pp. 309-323, 1973

[4] Wölfel, E., Nachgiebiger Verbund - Eine Näherungslösung und deren Anwendungsmöglichkeiten, Stahlbau, 6, pp. 173-180, 1987.

[5] Sagan, H., Introduction to the Calculus of Variations, Dover, New York, 1992.

[6] Girhammar, U.A., Gopu, V. K. A., Composite beam-columns with interlayer slip Exact analysis, J. of Struct. Engineering, 119, pp. 1265-1282, 1993.

[7] Beher, R.A., Minor, J.E., Norville, H.S., Structural behavior of architectural laminated glass, J. of Struct. Engineering, 119, pp. 202-222, 1993.

[8] Norville, H.S., King, K.W., Swofford, J.L., Behavior and strength of laminated glass, J. of Eng. Mechanics, 124, pp. 46-53, 1998.

[9] Bennison, S.J., Jagota, A., Smith, C.A., Fracture of glass/polyvinyl butyral laminates in biaxial flexure, J. of the Am. Cer. Society, 82, pp. 1761-1770, 1999.

[10] Bennison, S.J, Davies, P.S., Van Duser, A., Jagota, A., Structural performance of laminated safety glass made with "stiff" interlayers, Proceedings of Glass Performance Days, Tampere (Finland), 2001.

[11] Aşik, M.Z., Laminated glass plates: revealing of nonlinear behavior, Comp. and Structures, 81, pp. 2659-2671, 2003.

[12] Aşik, M.Z., Tezcan, S., A mathematical model for the behavior of laminated glass beams, Comp. and Structures, 83, pp. 1742-1753, 2005.

[13] Stephen, J.B., Sloan, J.G., Kristunas, D.F., Buehler, P.J., Amos, T., Smith, C.A., Laminated glass for blast mitigation: role of interlayer properties, Proceedings of Glass Performance Days, Tampere (Finland), 2005. 
[14] Ivanov, I.V., Analysis, modelling, and optimization of laminated glasses as plane beam, Int. J. of Sol. and Struct., 43, pp.6887-6907, 2006.

[15] Foraboschi, P., Behavior and failure strength of laminated glass beams, J. of Eng. Mechanics, 133, pp. 1290-1301, 2007.

[16] Bennison, S.J., Qin, M.H.X., Davies, P.S., High-performance laminated glass for structurally efficient glazing, Innovative Light-weight Structures and Sustainable Façades, Honk Hong, 2008.

[17] Bennison, S.J., Structural properties of laminated glass, Short Course, Glass Performance Days, Tampere, Finland, 2009.

[18] Calderone, I., Davies, P.S., Bennison, S.J., Huang, X. Gang, L., Effective laminate thickness for the design of laminated glass, Proceedings of Glass Performance Days, Tampere (Finland), 2009.

[19] Louter, C. Belis, J., Bos, F., Callewaert, D., Veer, F., Experimental investigation of the temperature effect on the structural response of SG-laminated reinforced glass beams, Eng. Structures, 32, pp. 1590-1599, 2010.

[20] ABAQUS, Analysis Users Manual, Version 6.10, Simulia. 